\title{
Prioritising smart factory investments - A project portfolio selection approach
}

Stephan Dreyer, Andreas Egger, Louis Püschel and Maximilian Röglinger

\begin{abstract}
Industry 4.0, which describes the transformation of existing production environments toward smart factories, is implemented in ever more manufacturing companies. Smart factories offer diverse advantages such as high flexibility, dynamic scheduling, as well as accurate fault diagnosis and prediction. Hence, manufacturing companies need support for assessing which projects they should implement to transform their production environment. As no such guidance exists in the literature, we propose a multi-dimensional decision model that accounts for interdependencies among production components, for projects with different performance effects, and for digital capabilities constitutive of smart factories (i.e., real-time ability, interoperability, virtualisation and decentralisation). The decision model schedules smart factory projects over multiple planning periods and assesses project roadmaps in line with objectives that comply with established performance measures and the digital capabilities of smart factories. We evaluate and discuss the decision model in interviews with two factory managers and three researchers with great experience in the smart factory domain. Based on a software prototype, we also successfully applied the decision model at a manufacturing company based on real-world data.
\end{abstract}

